\title{
Uncountable Graphs and Invariant Measures on the Set of Universal Countable Graphs
}

\author{
F. Petrov, A. Vershik
}

30.06 .09

\begin{abstract}
We give new examples and describe the complete lists of all measures on the set of countable homogeneous universal graphs and $K_{s^{-}}$ free homogeneous universal graphs (for $s \geq 3$ ) that are invariant with respect to the group of all permutations of the vertices. Such measures can be regarded as random graphs (respectively, random $K_{s}$-free graphs). The well-known example of Erdös-Rényi (ER) of the random graph corresponds to the Bernoulli measure on the set of adjacency matrices. For the case of the universal $K_{s}$-free graphs there were no previously known examples of the invariant measures on the space of such graphs.

The main idea of our construction is based on the new notions of measurable universal, and topologically universal graphs, which are interesting themselves. The realization of the construction can be regarded as two-step randomization for universal measurable graph : "randomization in vertices" and "randomization in edges". For $K_{s^{-}}$ free, $s \geq 3$ there is only randomization in vertices of the measurable graphs. The completeness of our lists is proved using the important theorem by D. Aldous about $S_{\infty}$-invariant matrices, which we reformulate in appropriate way.
\end{abstract}

\section{Contents}

1 Introduction: problem and results

1.1 Universal graphs .................... 2 
1.2 Random graphs and invariant measures on the set of the universal graphs . . . . . . . . . . . . . . . . . . . . . . . 4

1.3 How uncountable universal graphs can help toward countable ones: double randomization. . . . . . . . . . . . 5

1.4 About this paper. . . . . . . . . . . . . 6

2 Theme and variation on universal graphs 7

2.1 Countable graphs: the criterion of universality . . . . . . . . . 7

2.2 Universal measurable graphs . . . . . . . . . . . . . . . . . . . . . . . . . . .

2.3 Topologically universal graphs . . . . . . . . . . . . . . 11

3 Construction of continuous homogeneous graphs 13

4 Classification and the complete list of invariant measures on the set of universal graphs

4.1 Classification of invariant measures obtained by randomization in vertices ................ . . 18

4.2 Randomization in edges and description of the list of all invariant measures on the universal homogeneous $\left(K_{s}\right.$-free universal homogeneous) graphs. . . . . . . . . . . . . . . 19

4.2.1 The list of all invariant measures for the case of universal homogeneous graphs . . . . . . . . . . 20

4.2.2 The list of measures for $K_{s}$-free universal homogeneous graphs. . . . . . . . . . . . . . . 22

\section{Some problems and comments}

\section{Introduction: problem and results}

\section{$1.1 \quad$ Universal graphs}

Fix a countable set $V$ and consider the set $\mathcal{G}_{V}$ of all graphs (undirected, without loops and multiple edges) with $V$ as the set of vertices. Equip $\mathcal{G}_{V}$ with the weak topology (the base of the weak topology is formed by the collections of sets of graphs that have a given induced graph structure on a given finite set of vertices). The weak topology allows us to define the notion of Borel sets and Borel $\sigma$-field on $\mathcal{G}_{V}$, and to consider Borel probability measures on $\mathcal{G}_{\mathcal{V}}$. It is convenient to take the set of positive integers $\mathbb{N}$ as $V$. 
We can identify a graph $\Gamma$ with its adjacency matrix $A_{\Gamma}$ : an entry $e_{i, j}$, $i, j \in \mathbb{N}$, of $A_{\Gamma}$ is equal to 1 or 0 if $(i, j)$ is an edge or not an edge, respectively. Thus the space $\mathcal{G}_{\mathbb{N}}$ of graphs can be identified with the space $M_{\mathbb{N}}^{\text {Sym }}(0 ; 1)$ of all infinite symmetric zero-one matrices with zeros on the principal diagonal, equipped with the usual weak topology on the space of matrices.

The infinite symmetric group $\mathfrak{S}^{\mathbb{N}}$ of all permutations of the set $\mathbb{N}$ acts naturally on the space of graphs $\mathcal{G}_{\mathbb{N}}$. Each orbit of $\mathfrak{S}^{\mathbb{N}}$ is a class of isomorphic graphs, and the stabilizer of a given graph, as a subgroup of $\mathfrak{S}^{\mathbb{N}}$, is the group of all automorphisms of this graph. The action of $\mathfrak{S}^{\mathbb{N}}$ is continuous with respect to the weak topology on $\mathcal{G}_{\mathbb{N}}$, and to the weak topology on the group $\mathfrak{S}^{\mathbb{N}}$ itself. In terms of the space of matrices $M_{\mathbb{N}}^{\text {Sym }}(0 ; 1)$, this action obviously means a simultaneous permutation of the rows and columns of the adjacency matrices. The action naturally extends to an action on Borel measures on the spaces of graphs and matrices.

We will consider subsets of $\mathcal{G}_{\mathbb{N}}$ that are invariant under the action of $\mathfrak{S}^{\mathbb{N}}$, and invariant Borel probability measures on such sets. Of most interest are subsets of $\mathcal{G}_{\mathbb{N}}$ on which the group $\mathfrak{S}^{\mathbb{N}}$ acts transitively; namely, an example important for our purposes is the family of universal graphs in a category of graphs.

Consider a small category $\mathcal{C}$ whose objects are finite or countable graphs (the sets of vertices of these graphs are subsets of $\mathbb{N}$ ) that contains a universal object. This means that there is an object of $\mathcal{C}$, a countable graph $\Gamma$, that satisfies the following properties:

1) $\Gamma$ contains any finite graph of the category $\mathcal{C}$ as a subobject (up to isomorphism)

and

2) the group of all isomorphisms of $\Gamma$ acts transitively on the set of isomorphic finite subgraphs of $\Gamma$.

Such graphs are called homogeneous universal graphs of the category $\mathcal{C}$. Hereafter we just call them "universal", without explicit mentioning homogeneity. Fraïssé's theory (see, e.g., [9]) gives transparent necessary and sufficient conditions for the description of categories that have a universal graph. We may assume that the sets of vertices of all universal graphs in all these categories coincide with the whole set $\mathbb{N}$, so we can identify graphs with their adjacency matrices from $M_{\mathbb{N}}^{S y m}(0,1)$, and the set of universal graphs is an orbit of the action of the group $\mathfrak{S}^{\mathbb{N}}$. By a "random graph" in a given category we mean a $\mathfrak{S}^{\mathbb{N}}$-invariant Borel probability measure on the set of graphs that is concentrated on the set of universal graphs of this category. 
Here we restrict ourselves to the following category: $\mathcal{C}_{s}, s>2$, is the category of all finite or countable graphs that contain no s-cliques $K_{s}$ (an $s$-clique is a complete graph with $s$ vertices, $s>2$ ). Also denote by $\mathcal{C}$ the category of all finite or countable graphs. It is well known that Fraïssé's axioms are valid in these cases, and there are universal graphs in all these categories. A corollary of the existence of universal graphs asserts that all universal graphs are mutually isomorphic, so a universal graph is unique up to isomorphism; consequently, the set of all universal graphs is an orbit of the group $\mathfrak{S}^{\mathbb{N}}$. We describe the set of invariant measures on these orbits.

\subsection{Random graphs and invariant measures on the set of the universal graphs}

We consider a "random $K_{s}$-free graph," which is the same as a $\mathfrak{S}^{\mathbb{N}}$-invariant measure on the set of universal $K_{s}$-free graphs. The existence of a $\mathfrak{S}^{\mathbb{N}}$ invariant measure on the set of ordinary universal graphs (the category $\mathcal{C}$ ) is well known: this is the Erdös-Rényi [7] random graph. In our terms, the examples of Erdös and Rényi are the Bernoulli measures on the space $M_{\mathbb{N}}^{\text {Sym }}(0,1)$ of adjacency matrices with the distribution $(p, 1-p), 0<p<1$, for each entry. Note that for $p=1 / 2$ this Bernoulli measure is the weak limit of the uniform measures on the sets of finite graphs with $n$ vertices as $n$ tends to infinity. We will see that there are many other $\mathfrak{S}^{\mathbb{N}}$-invariant measures on the set of universal graphs.

As to the categories $\mathcal{C}_{s}, s>2$, no invariant measures (or no random graphs) were known at all. For the case $s=3$, it was known that the weak limit of the uniform measures on the set of finite triangle-free graphs with $n$ vertices as $n$ tends to infinity is not a measure on the set of universal triangle-free graphs, but an invariant measure on the set of universal bipartite graphs. This follows from the results of [6, 11] on asymptotic estimations of the number of odd cycles in typical triangle-free graph 1 . This means that the uniform measure, as an approximation tool, is too rough for obtaining the desired measure. Nevertheless we proved that there exist uncountably many invariant ergodic measures on the set of $K_{s}$-free graphs for $s>2$. Note the paradoxical fact that, in spite of the transitivity of the action of the group $\mathfrak{S}^{V}$ on the set of universal graphs, there exist uncountably many different (pairwise singular) $\mathfrak{S}^{\mathbb{N}}$-invariant ergodic measures; this is a new

\footnotetext{
${ }^{1}$ We are grateful to Professor G. Cherlin for the references to these papers.
} 
manifestation of Kolmogorov's effect, see details in [18].

Remark that our construction of the universal continuous graph for the case $s=2,3$ is shift invariant which means that there is a transitive action of a group $\mathbb{R}$ on the set of vertices (which is $\mathbb{R}$ ) of the continuous graph. For the countable universal graph the existence of the transitive action of the group $\mathbb{Z}$ on the set of vertices is trivial; for the case of triangle free universal graph it was proved by C. Henson [8]; who also had proved nonexistence of such action for $s>3$. 2 We also mentioned that fact for continuous case.

\subsection{How uncountable universal graphs can help toward countable ones: double randomization.}

For constructing $\mathfrak{S}^{V}$-invariant measures on the space of universal graphs, we will use a very natural general method of constructing invariant measures on the set of infinite matrices. It looks like the Monte-Carlo or randomization method. Specifically, we take a continuous graph, that is, a standard measure space $(X, m)$, regarded as the set of vertices, and a subset $E \subset X^{2}$, regarded as the set of edges, and then choose vertices (points of $X$ ) at random, independently, with distribution $m$; the induced countable subgraph is our random graph. If we want to obtain an invariant measure on the set of universal ( $K_{s}$-free universal, etc.) graphs, we must use (and first define!) a universal (respectively, $K_{s}$-free universal, etc.) continuous graph. Thus our examples of invariant measures on the space of universal graphs come from "randomization in vertices" of universal continuous measurable graphs 3 Note that the notion of a universal continuous graph is perhaps of interest in itself in the theory of models and "continuous combinatorics." It looks similar to the universal Urysohn space if we compare it with the countable universal metric space. We will consider this analogy in a separate paper 4

The method of the randomization of the vertices does not give all invariant measures on the set of universal graphs (or on the set of all countable graphs). Even Erdos-Renji example of random graph is not of that type. In order to describe all invariant measures on the set of universal graphs

\footnotetext{
${ }^{2}$ We are grateful to the reviewer who pointed out to this paper.

${ }^{3}$ Note that our notion of universality of continuous graphs is not a categorical universality and homogeneity.

${ }^{4}$ The notion of a continuous graph in general must be very useful in variational calculus, geometrical optimal control, etc.
} 
as well as invariant measures on the other sets of the countable graphs, we must generalize this method and use another kind of randomization, namely, "randomization in edges". In this paper we shortly describe this construction based on the notion of generalized graph and on the important theorem due to D. Aldous [1, which describes in some sense all $\mathfrak{S}^{\mathbb{N}}$-invariant measures on the space of the infinite matrices. In particulary we apply this construction for the universal graphs. It gives the list of all $\mathfrak{S}^{\mathbb{N}}$-invariant ergodic measures on the set of universal or $K_{s}$-universal graphs.

Remark that in order to prove that our constructions exhaust the list of all invariant measures on the set of universal ( $K_{s}$-free universal) graphs we use the important theorem due to D. Aldous [1] about invariant measures on the space of matrices. We formulate that theorem in a suitable version, which will be considered with a new proof of it by the second author in the separate place. See also [16]), where these problems are linked to the problem of classification of measurable functions of the several variables.

Thus our scheme looks like the following transitions: universal Borel graph with measures $\rightarrow$ topologically universal graph $(\rightarrow$ homogeneous topologically universal graph) ${ }^{5} \rightarrow$ randomization in vertices $\rightarrow$ invariant measures on the set of countable universal graphs $\rightarrow$ randomization in edges $\rightarrow$ the list of all invariant measures on the set of countable universal $\left(K_{s}\right.$-free universal) graphs. In brief, our description of invariant measures reduces to the choice of a deterministic continuous graph, then to randomization of its vertices (randomly choosing some vertices), and then to randomization of edges.

The method of this paper does not help to solve the problem due to Prof. G. Cherlin about existence of the finite triangle free "almost" universal graph. The reason is that it is difficult to extract from our constructions the implicit type of finite dimensional approximations of the constructed invariant measures. In the same time the continuous models for constructions of random countable objects can be applied in many situations.

\subsection{About this paper.}

Let us give a short description of the contents of the paper. In the second section we consider the notions of continuous graphs and universal continuous graphs of various types using a generalization of the criterion of universality. We give two kinds of definitions: for measurable (Borel) graphs and topolog-

\footnotetext{
${ }^{5}$ The homogeneity is used for the ordinary and triangle-free cases only.
} 
ical graphs; the latter ones are more convenient for our goals. Section 3 is devoted to a particular construction of topologically universal $\left(K_{s}\right.$-free universal) graphs. We define even a shift-invariant graph structure with $\mathbb{R}$ as the set of vertices for the ordinary and triangle-free cases. This gives the existence of nontrivial $\mathfrak{S}^{\mathbb{N}}$-invariant measures on the set of universal graphs. The main part of the Section 4 has deal with the general constructions of the invariant measures not only for universal graphs. We give the classification of measures obtained in terms of the randomization in edges in the spirit of paper [16]. Then we define the generalized graph and give a general scheme of the double randomization of the universal continuous graphs (in vertices and edges). This is tightly connected with the mentioned above Aldous's theorem about the list of all $\mathfrak{S}_{\mathbb{N}}$-invariant measures on the $0-1$ matrices. This gives a list of all such invariant measures for universal and $K_{s}$-free $(s>2)$ universal graphs. Some problems and comments are collected in the last section. One of the main practical problems is to find directly the finite-dimensional distributions of our measures on the set of universal graphs, or, more specifically, to describe the approximation of random universal graphs in our sense in terms of random finite graphs.

Professor T. Tao informed the second author that the idea of using continuous graphs has already appeared in the recent papers by L. Lovasz and his coauthors [12, 13, where an analog of a continuous weighted graph was defined. In [5], this notion was also associated with Aldous' theorem. Our goals and constructions are different from those constructions: we consider universal continuous graphs.

The authors are grateful to Professors N. Alon, G. Cherlin and T. Tao for important references, the reviewer of the paper for very useful comments and to Prof. N. Tsilevich for her help with preparing the final version of the paper and useful criticism.

\section{Theme and variation on universal graphs}

\subsection{Countable graphs: the criterion of universality}

Recall that the universality of a countable graph $\Gamma_{u}$ is equivalent to the following two conditions:

( $i$ ) any finite graph $\gamma$ can be isomorphically embedded into $\Gamma_{u}$;

(ii) for any two isomorphic finite induced subgraphs $\gamma_{1}, \gamma_{2}$ of $\Gamma_{u}$, any 
isomorphism between them can be extended to an isomorphism of the whole graph $\Gamma_{u}$.

It is easy to prove that the following well-known criterion is equivalent to (i)\&(ii) (see, e.g., [3]):

(iii) for any two disjoint finite subgraphs $\gamma_{1} \subset \Gamma_{u}$ (call it "black") and $\gamma_{2} \subset \Gamma_{u}$ (call it "white") there exists a vertex $v \in \Gamma_{u}$ that is joined with the white vertices and is not joined with the black ones.

Now we will give an analog of this condition for the case of triangle-free and $K_{s}$-free graphs.

Theorem 1. 1. A countable triangle-free graph $\Gamma$ is a universal triangle-free graph if and only if the following condition is satisfied:

$\left(\right.$ iii $\left._{3}\right)$ for any two disjoint finite subgraphs $\gamma_{1} \subset \Gamma_{u}$ (call it "black") and $\gamma_{2} \subset \Gamma_{u}$ (call it "white"), where the white subgraph has no edges, there exists a vertex $v \in \Gamma_{u}$ that is joined with all white vertices and is not joined with the black vertices.

2. For $s>2$, a countable $K_{s}$-free graph $\Gamma$ is a universal $K_{s}$-free graph if and only if the following condition is satisfied:

$\left(\right.$ iii $\left.i_{m}\right)$ For any two disjoint finite subgraphs $\gamma_{1} \subset \Gamma_{u}$ (call it "black") and $\gamma_{2} \subset \Gamma_{u}$ (call it "white"), where the white subgraph is $K_{s-1}$-free, there exists a vertex $v \in \Gamma_{u}$ that is joined with all white vertices and is not joined with the black vertices.

Of course, the first part of the theorem is a special case of the second one, and in what follows we will consider the triangle-free case as a special case of $K_{s}$-free graphs with $s=3$. The proof of the theorem is a simple modification of the proof of the previous theorem.

\subsection{Universal measurable graphs}

Now we give the definition of Borel (measurable), topologically universal, and topologically universal $K_{s}$-free graphs for $s>2$. But first of all we will give the definition of continuous graphs themselves. Our definitions of these notions are not of the greatest possible generality, but they are appropriate for our goals.

Recall that a standard (uncountable) Borel space $X$ is a space with a fixed $\sigma$-field of subsets that is Borel isomorphic to the interval $[0,1]$ equipped with the $\sigma$-field of Borel subsets. 
Definition 1. A Borel (undirected) graph is a pair $(X, E)$ where $X$ is a standard Borel space and $E \subset X \times X$ is a symmetric Borel subset in $X \times X$ that is disjoint from the diagonal $\{(x, x), x \in X\}$.

We will denote $E_{x}=\{y \in X:(x, y) \in X\}$ and $E_{x}^{\prime}=X \backslash E_{x}$. Note that if $\left\{x_{k}\right\}_{k=1}^{\infty}$ is a finite or countable sequence in $X$, then it can be regarded as an ordinary finite or countable subgraph of $(X, B)$ with the induced graph structure. We say that a Borel graph is pure if $E_{x} \neq E_{y}$ for $x \neq y$. Note that universal countable graphs are pure.

The following measure-theoretic definition is more useful for us.

Definition 2. A measurable (Borel) graph is a triple $(X, m, E)$ where $(X, m)$ is a standard Lebesgue space with a continuous probability measure $m$ (i.e., the pair $(X, m)$ is isomorphic in the measure-theoretic sense to the interval $[0,1]$ equipped with the Lebesgue measure) and $E \subset X \times X$ is a symmetric measurable set of positive $(m \times m)$-measure 6

A measurable graph is called pure if the measurable map $x \rightarrow E_{x}(\bmod 0)$ from $X$ to sigma algebra of mod0-classes of measurable sets is injective mod 0 .

Definition 3. A universal (respectively, $K_{s}$-free universal) measurable graph is a pure measurable graph $(X, m, E)$ that satisfies the following property: for almost all sequences $\left\{x_{k}\right\}_{k=1}^{\infty} \in X^{\infty}$ with respect to the Bernoulli measure $m^{\infty}$ in the space $X^{\infty}$, the induced countable graph on the set of vertices $\left\{x_{k}\right\}$ is a universal countable graph (respectively, a $K_{s}$-free universal countable graph). 7

The definition above is indirect, but it is not difficult to formulate direct definition which is equivalent to the previous.

Theorem 2. 1. The pure measurable graph $(X, m, E)$ is universal in the above sense iff for almost any two disjoint finite sets $\left\{x_{1}, \ldots, x_{n}\right\} \in X$ and $\left\{y_{1}, \ldots, y_{m}\right\} \in X$ the $m$-measures of the following intersections:

$$
m\left(\bigcap_{i, j}\left(E_{x_{i}} \cap E_{y_{j}}^{\prime}\right)\right)
$$

\footnotetext{
${ }^{6}$ Strictly speaking, we must consider the class of sets that are equal to $E$ up to a set of zero measure; consequently, we define a class of $(\bmod 0)$-coinciding graphs.

${ }^{7}$ It is more correct to call such graphs countably universal, because the condition deals only with countable subsets of $X$.
} 
are positive; 2 . The pure measurable graph $(X, m, E)$ is $K_{s}$-free universal iff there are almost no s-tuples in $X$ for which the induced (by the set $E$ ) graph is a $K_{s}$-graph; and for any positive integers $k$, $t$ and for almost any two finite subsets $x=\left\{x_{1}, \ldots, x_{k}\right\},\left\{y_{1}, \ldots, y_{t}\right\} \subset X$ such that the induced graph $x$ has no $K_{s-1}$-subgraphs, the m-measure of the following intersection is positive:

$$
m\left(\bigcap_{i, j}\left(E_{x_{i}} \cap E_{y_{j}}^{\prime}\right)\right)>0 .
$$

For $s=3$ this gives the definition of a triangle-free topologically universal graph.

The proof of the equivalence consists in direct application of the ergodic theorem (or even individual law of large numbers) to the indicators of intersections defined above.

A direct corollary of our definition is the following theorem which will be used in what follows.

Theorem 3 (Construction of invariant measures). Let $(X, m, E)$ be a universal (respectively, $K_{s}$-free universal) measurable graph. Consider the space

$$
X^{\infty}=\prod_{n=1}^{\infty}(X, m)
$$

and the map

$$
\begin{gathered}
F: X^{\infty} \rightarrow M_{\mathbb{N}}(0,1), \\
F\left(\left\{x_{i}\right\}\right)=\left\{e_{i, j}\right\}, \quad e_{i, j}=\chi_{E}\left(x_{i}, x_{j}\right), \quad i, j \in \mathbb{N},
\end{gathered}
$$

where $\chi_{E}$ is the characteristic function of the set $E \subset X \times X$. Denote by $F^{*}$ the map defined on the space of Borel measures on $X^{\infty}$ by the following formula: if $\alpha$ is a Borel measure on $X^{\infty}$, then $\left[F^{*}(\alpha)\right](C)=\alpha\left(F^{(-1)}(C)\right.$ ), $C \subset M_{\mathbb{N}}(0,1)$. Then the measure $F^{*}\left(m^{\infty}\right) \equiv \mu_{\{X, m, E\}}$ is an $\mathfrak{S}_{\mathbb{N}}$-invariant measure on the set of universal (respectively, $K_{s}$-free universal) homogeneous countable graphs.

Proof. Follows from the fact that the Bernoulli measure $m^{\infty}$ is $\mathfrak{S}^{\mathbb{N}}$-invariant.

The following formula gives the implicit expression of the measure of cylindric sets (it does not use the condition of universality). Suppose $A=$ 
$\left\{a_{i, j}\right\}, i, j=1 \ldots n$ is $(0-1)$-matrix of order $n$, and $C_{A}$ is a cylindric set of all infinite $(0-1)$-matrices, which has the matrix $A$ as submatrix on the NW-corner. Then the value of the measure $\mu_{\{X, m, E\}} \equiv \mu$ on the cylinder $C_{A}$ is

$\mu\left(C_{A}\right)=m^{n}\left\{\left(x_{1}, x_{2} \ldots x_{n}\right):\left(x_{i}, x_{j}\right) \in E ; \quad\right.$ if $\quad a_{i, j}=1 ; \quad\left(x_{k}, x_{r}\right) \notin E \quad$ if $\left.\quad a_{k, r}=0\right\}$

where $m^{n}=m \times \ldots(n) \cdots \times m$

Because the given criterion of the universality is difficult to check for measurable graphs, we will use topological version of universality which is much more convenient.

\subsection{Topologically universal graphs}

As we have mentioned, it is not easy to check that a given measurable graph $(X, m, E)$ is a universal measurable graph. For this reason, we will give a more restrictive definition of topological universality, whose conditions are easier to check.

Let us define a topologically universal graph. For simplicity, we assume that $X$ is a Polish (= metric separable complete) space, but this is not necessary.

Given a set $Y \subset X$, denote its complement by $Y^{\prime}=X \backslash Y$ and its closure by $\bar{Y}$. A topological graph (undirected, without loops) is a pair $(X, E)$ where $X$ is a Polish space and $E \subset X \times X$ is a closed subset that has a nonempty interior and an empty intersection with the diagonal: 8 Put $E_{x}=\{y \in X$ : $(x, y) \in X\}$. We say that a topological graph is pure if $E_{x} \neq E_{y}$ for $x \neq y$.

Definition 4. 1. A pure topological graph $(X, E)$ is called topologically universal if the set $E$ satisfies the following property:

$(U)$ For any two disjoint finite sets $\left\{x_{1}, \ldots, x_{n}\right\} \in X$ and $\left\{y_{1}, \ldots, y_{m}\right\} \in$ $X$, the intersection

$$
\bigcap_{i, j}\left(E_{x_{i}} \cap E_{y_{j}}^{\prime}\right)
$$

has a nonempty interior.

\footnotetext{
${ }^{8}$ Our definition allows vertices to have uncountably many neighbors. There are many other definitions of topological graphs and topological graphs with weights; one of them uses the notion of a polymorphism or Markov transformation.
} 
2. A topological graph $(X, E)$ is called topologically $K_{s}$-free universal if $\left(U_{m}\right)$ there are no $m$-tuples in $X$ for which the induced (by the set $E$ ) graph is a $K_{s}$-graph (a complete $m$-graph); and for any positive integers $k, t$ and any two finite subsets $x=\left\{x_{1}, \ldots, x_{k}\right\},\left\{y_{1}, \ldots, y_{t}\right\} \subset X$ such that the induced graph on $x$ has no $K_{s-1}$-subgraphs, the set

$$
\bigcap_{i, j}\left(E_{x_{i}} \cap E_{y_{j}}^{\prime}\right)
$$

has a nonempty interior in $X$.

For $s=3$ this gives the definition of a triangle-free topologically universal graph.

It is worth mentioning that a topologically universal graph is not a "universal topological graph" in the sense of the category of topological graphs; our definition is more flexible. As in the case of measurable graphs, it is more correct to call it a "countably universal topological graph."

Recall that a Borel measure on a Polish space is called nondegenerate if it is positive on all nonempty open sets.

Theorem 4. Let $(X, E)$ be a topologically universal graph (respectively, a topologically universal $K_{s}$-free graph, $\left.s>2\right)$; then for every nondegenerate Borel probability measure $m$ on the space $X$, the triple $(X, E, m)$ is a universal measurable (respectively, universal measurable $K_{s}$-free) graph in the sense of the definition of Section 2.2.

Proof. Let $m$ be a nondegenerate measure on $X$. We must check that the property $(i i i)$ (respectively, $\left(i i i_{M}\right)$ ) from Section 2.1 is valid for almost all (with respect to the Bernoulli measure $m^{\infty}$ ) sequences $\left\{x_{k}\right\}$. First of all, almost all sequences $\left\{x_{k}\right\}$ are everywhere dense in the separable metric space $X$. Consequently, every such sequence $\left\{x_{k}\right\}$ contains points from any open set in $X$. Since all the sets $\cap_{i, j} E_{\ldots} \cap E_{\ldots}^{\prime}$, described in Definition 4 , have a nonempty interior, the proof is done. The measurable graph is pure since the topologically universal graph is pure.

Using this theorem, we immediately obtain the following corollary, which shows how to produce required measures on the set of universal graphs.

Corollary 1. For every nondegenerate measure $m$ on a topologically universal (respectively, triangle free, $K_{s}$-free) graph $(X, E)$, the measure $\mu_{\{X, m, E\}}$ is a $\mathfrak{S}^{\mathbb{N}}$-invariant measure on the set of universal (respectively, universal triangle-free, universal $K_{s}$-free) countable graphs. 
The existence of topologically universal graphs and topologically universal $K_{s}$-free graphs is proved in the next section.

The reason why we introduce, in addition to the notion of a measurable universal graph, the notion of a topologically universal graph is that it is difficult to formulate a measure-theoretic analog of the property that the interiors of the sets $E(x, y)$ are nonempty, or equivalent properties, which are important for extending a countable graph structure to a continuous one. But there are no doubts that this notion is useful in itself.

Remark 1. All previous definitions can be written in a more rigid form if we use the invariance with respect to an action of a group on the set of vertices of the graph. Let $G$ be a group, and let the set of vertices $X$ be a $G$-space; we can repeat our definitions of continuous and universal graphs for a $G$ invariant graph structure. For example, let $X=G$, and let the set of edges $E \subset G \times G$ be left $G$-invariant: $E=\left\{(g, h): g^{-1} h \in Z\right\}$, where $Z \subset G$. Group-invariant graph structures (Cayley objects in the terminology of [3]) were considered in [3, 4, 19].

\section{Construction of continuous homogeneous graphs}

We will prove the existence of topologically universal graphs and topologically universal $K_{s}$-free graphs. According to the previous results, our construction gives examples of invariant measures on the space of universal graphs. As we will see, there are many such constructions which produce uncountably many nonequivalent invariant measures. We choose the simplest example, namely, consider the additive group $X=\mathbb{R}$ as the set of vertices of a topological graph and define an appropriate set of edges (a subset of $\mathbb{R}^{2}$ ). Moreover, for the case of ordinary universal graphs and triangle-free graphs, we suggest a graph structure that is shift-invariant:

$$
E=\{(x, y):|x-y| \in Z\} \subset \mathbb{R}^{2},
$$

where the set $Z \subset(0,+\infty)$ will be constructed by induction. This means that the additive group $\mathbb{R}$ acts by the automorphisms transitively on the set of vertices.

We will prove the following main result. 
Theorem 5. 1. There is a topologically universal graph (respectively, topologically universal triangle-free graph) with the additive group $\mathbb{R}$ as the set of vertices and a shift-invariant graph structure.

2. There is a topologically universal $K_{s}$-free graph (for $s>3$ ) with the additive group $\mathbb{R}$ as the set of vertices. There is no universal $K_{s}$-free graph for $s>3$ with a shift-invariant graph structure.

Proof. 1. We will begin with the construction of a countable universal (triangle-free universal) graph with the additive group of rational numbers $\mathbb{Q}$ as the set of vertices and a shift-invariant graph structure. After that we extend the construction onto $\mathbb{R}$.

We choose $X=\mathbb{Q}$ as the set of vertices and construct a set $Z \subset \mathbb{Q}$ that will be the subset of vertices joined by edges with zero $\mathbf{0} \in \mathbb{Q}$. Thus $(x, y)$ is an edge of our graph if and only if $|x-y| \in Z, x \neq y$. We construct $Z$ as the union of disjoint nondegenerate intervals of $\mathbb{Q}$ with rational endpoints such that any bounded set $M \subset \mathbb{R}$ contains only finitely many such intervals. The required shift-invariant structure of a continuous universal (triangle-free universal) graph on $\mathbb{R}$ will appear if $\bar{Z}$, the closure of $Z$, is the set of vertices $x \in \mathbb{R}$ that are joined by edges with $\mathbf{0} \in \mathbb{Q}$. In a sense, it is a completion of that universal (triangle-free universal) rational graph.

It is easy to reformulate the conditions of universality in terms of the set $Z \subset \mathbb{R}_{+}$using the shift-invariance:

For a universal graph, we obtain the following condition.

$(U)$ For every pair of disjoint finite sets of rational numbers $\left\{x_{1}, \ldots, x_{k}\right\}$, $\left\{y_{1}, \ldots, y_{t}\right\}$ there exists a rational number $c$ such that $\left|c-x_{i}\right| \in Z, i=$ $1,2, \ldots, k ;\left|c-y_{j}\right| \notin Z, j=1,2, \ldots, t$.

For a universal triangle-free graph, the condition on the set $Z$ is more rigid:

$(U T F)$ (a) The sum-free condition: The equation $x+y=z$ has no solutions with $x, y, z \in \bar{Z}$ (this is a corollary of the triangle-free condition for graphs).

(b) for every pair of disjoint finite sets of rational numbers $\left\{x_{1}, \ldots, x_{k}\right\}$, $\left\{y_{1}, \ldots, y_{t}\right\}$ such that $\left|x_{i}-x_{j}\right| \notin \bar{Z}, 1 \leq i<j \leq k$, there exists a rational number $c$ such that $\left|c-x_{i}\right| \in Z, i=1,2, \ldots, k ;\left|c-y_{j}\right| \notin Z, j=1,2, \ldots, t$.

In both cases, our construction will satisfy a stronger condition, which is necessary for our purposes: there exists an interval $\left(c_{1}, c_{2}\right)$ of points $c$ satisfying the above property. 
The construction of the set $Z$ is inductive and based on the enumeration of arrays of points from $\mathbb{Q}$. We will use the simplest method of enumeration suitable both for ordinary and triangle-free graphs.

Choose $\gamma$, a pair of finite sets of disjoint intervals; the first set

$$
\left\{\left(a_{1}, a_{1}^{\prime}\right),\left(a_{2}, a_{2}^{\prime}\right), \ldots,\left(a_{k}, a_{k}^{\prime}\right)\right\}=\gamma^{a}
$$

of the pair will be called "white," and the second set

$$
\left\{\left(b_{1}, b_{1}^{\prime}\right),\left(b_{2}, b_{2}^{\prime}\right), \ldots,\left(b_{\ell}, b_{\ell}^{\prime}\right)\right\}=\gamma^{b}
$$

will be called "black"; all the closures of these $k+\ell$ intervals are mutually disjoint. We will call such a pair $\gamma$ a pattern. In the triangle free case require that the closure of white part of the pattern is sum-free (i.e. consider only such patterns). There are countably many patterns, so we can label all patterns with positive integers $\gamma_{1}, \gamma_{2}, \ldots$. Note that for every pair $\bar{x}=$ $\left(x_{1}, \ldots, x_{p}\right), \bar{y}=\left(y_{1}, \ldots, y_{q}\right)$ of disjoint finite subsets of $\mathbb{Q}$, there exists a pattern whose white part contains the set $\bar{x}$ and black part contains the set $\bar{y}$.

For each pattern $\gamma_{n}$ we will define by induction a set $Z_{n}$, the union of finitely many intervals with rational endpoints, such that for all $z \in Z_{n}$, $u \in Z_{n+1}$ we have $z<u$ (the monotonicity condition). Then $Z$ will be the union of these $Z_{n}: Z=\cup_{n} Z_{n}$.

As the induction base we can take an arbitrary pattern, or even empty set.

Now we will consider two cases.

1) Construction of a universal continuous graph.

Assume that we have already constructed sets $Z_{1}, \ldots, Z_{n-1}$, each of which is the union of closed disjoint intervals and satisfies the monotonicity condition above. Assume also that the condition $(U)$ is satisfied for all patterns with numbers less than $n-1$. More exactly, if a set $\bar{x}=\left(x_{1}, \ldots, x_{k}\right)$ belongs to the white part of a pattern with number less than $n-1$ and a set $\bar{y}=\left(y_{1}, \ldots, y_{t}\right)$ belongs to the black part of this pattern, then there exists an open interval $C \subset \mathbb{Q}$ such that $\left|c-x_{i}\right| \in \cup_{1}^{n-1} Z_{i}$ and $\left|c-y_{j}\right| \notin \cup_{1}^{n-1} Z_{i}$ for every $c \in C$. Consider the next pattern $\gamma_{n}=\left(\gamma^{a}=\cup_{1}^{k}\left(a_{i}, a_{i}^{\prime}\right), \gamma^{b}=\cup_{1}^{s}\left(b_{j}, b_{j}^{\prime}\right)\right)$ and define a set $Z_{n}$ as follows. Find such a large $c$ that

$$
c>\max _{i, j}\left\{a_{i}, b_{j}\right\}+\max \left\{z: z \in \cup_{i=1}^{n-1} Z_{i}\right\}+1,
$$


and put $Z_{n}=\cup_{i=1}^{k}\left(c-a_{i}^{\prime}-\varepsilon, c-a_{i}+\varepsilon\right)$. It is clear that for small enough $\varepsilon$ all points that belong to a sufficiently small neighborhood of $c$ are joined by edges with the white part of $\gamma_{n}$ and are not joined with the black part of $\gamma_{n}$, because the shifted segments $\left[c-a_{i}^{\prime}, c-a_{i}\right]$ and $\left[c-b_{j}^{\prime}, c-b_{j}\right] i=1, \ldots, k$, $j=1, \ldots, s$, are disjoint, and so their small neighborhoods are disjoint as well. This completes the construction of the set $Z=\cup_{n} Z_{n}$. Now let us check that the graph with the set of vertices $\mathbb{Q}$ and the edges $\{(x, y):|x-y| \in Z\}$ is a universal countable graph. It suffices to mention that for every pair $\bar{x}=\left(x_{1}, \ldots, x_{l}\right), \bar{y}=\left(y_{1}, \ldots, y_{p}\right)$ of finite sets from $\mathbb{Q}$ there exists a pattern whose white part contains $\bar{x}$ and black part contains $\bar{y}$. Finally, consider the closure $\bar{Z}$ of the set $Z$ in $\mathbb{R}$. We must prove that the graph with $\mathbb{R}$ as the set of vertices and $\{(x, y): x, y \in \mathbb{R},|x-y| \in \bar{Z}\}$ as the set of edges is a universal graph. Choose a pair $\bar{x}=\left(x_{1}, \ldots, x_{l}\right), \bar{y}=\left(y_{1}, \ldots, y_{p}\right)$ of finite sets from $\mathbb{R}$ and find a pattern $\gamma$ whose white part contains $\bar{x}$ and black part contains $\bar{y}$. The shift-invariance of the graph structure follows from the construction.

2) In the case of a triangle-free graph we have only one additional remark to our construction. As we have mentioned, the graph defined in the induction base contains no triangles; and, by the induction hypothesis, no triangles appear when we define the sets $Z_{i}, i<n$. Let us check that no triangles appear when we define the set $Z_{n}$. Recall that we must consider only the white part of the pattern, because the point $c$ is not joined by edges with the black part. But the slightly enlarged white part has no edges by hypothesis, so the new edges do not produce triangles. As before, the extension of the graph structure onto $\mathbb{R}$ is defined by the closure $\bar{Z}$ of the set $Z$; since we have chosen a sufficiently small open neighborhood of the point $c$, the continuous graph inherits the absence of triangles.

2. Now consider the case of a $K_{s}$-free universal countable graph for $s>$ 3. The existence of a universal countable graph is a corollary of Fraïssé's axioms (one needs to check only the amalgamation axiom, see [9]). But even in the countable case for $s>3$ there is no universal shift-invariant graph structure. More exactly, for a $K_{s}$-free universal graph there is no transitive action preserving graph-structure of the group $\mathbb{Z}$ on its vertices. Consider the case $s=4$. Assume that a shift-invariant universal $K_{4}$-free graph on the group $\mathbb{Z}$ does exist. Let $(0, a)$ be an edge. Choose $b$ such that $(b, 0)$ is an edge, but $(b, a)$ and $(b,-a)$ are not (this is possible by the universality). Then $(0, a+b)$ is not an edge (as well as $(b,-a))$, and hence the quadruple $(0, a, b, a+b)$ does not contain triangles (it is a quadrangle without diagonals). Hence, again by the universality, there exists $x$ joined 
with all points $0, a, b, a+b$. Then it is easy to check that the set $(0, a, x, x-b)$ is a 4 -clique. We obtain a contradiction. In the case $s>4$ a contradiction may be obtained in a similar way, just start not from the edge $(0, a)$, but from some $(s-2)$-clique.

The same claim is still true if we replace the group $\mathbb{Z}$ with an arbitrary abelian group.

But the problem of constructing a universal continuous $K_{s}$-free graph for $s>3$ without the requirement of shift-invariance is very easy.

Let the set of vertices be $\mathbb{R}$. Again we define a pattern as a set of disjoint intervals with rational endpoints colored black and white. Let us enumerate all patterns as above. We will construct by induction a symmetric closed set $Z \subset \mathbb{R} \times \mathbb{R}$ with a nonempty interior, which will be the set of edges of our graph. As the induction base, we can choose $Z_{1}$ to be some square $[a, 2 a] \times[a, 2 a], 0<a$. At the $n$th step we consider the $n$th pattern $\gamma$ and fix the restriction of the set $\cup_{i=1}^{n-1} Z_{i}$ to the subgraph induced by the large segment $\left[-M_{k}, M_{k}\right]$, where $M_{n}=\max \left\{x: x \in \gamma_{n} \cup\left(-\gamma_{n}\right)\right\}+M_{n-1}+n+1$. Next we check whether there are cliques of size $n-1$ with white vertices of $\gamma_{n}$. If there are such cliques, we replace $n$ by $n+1$. If there are no such cliques, we add to the set $\cup_{i=1}^{n-1} Z_{i}$ the set $\left[M_{n}+1, M_{n}+2\right] \times \gamma_{n}^{w}$ (where $\gamma_{n}^{w}$ stands for the white part of $\gamma_{n}$ ) and then symmetrize it in $\mathbb{R} \times \mathbb{R}$. It is easy to check that after considering all patterns we get a topologically universal $K_{s}$-free graph.

We have proved that required topological and measurable universal continuous $K_{s}$-free graphs do exist, and this gives us examples of $\mathfrak{S}^{\mathbb{N}}$-invariant measures on the space of adjacency matrices.

The question which was posed in the first version of this paper whether it is possible to construct a group-invariant structure of a topologically universal graph for the compact group instead or $\mathbb{R}$ has an easy negative answer as the reviewer of the paper had mentioned.

Note that the concrete examples of $\mathfrak{S}^{\mathbb{N}}$-invariant measures on the set of universal countable graphs that we have obtained here are new and different from the Erdös-Rényi examples. For the construction we use Theorems 3 and 4:

Let

$$
d m(t)=\frac{1}{\sqrt{2 \pi}} \exp \left\{-\frac{t^{2}}{2}\right\} d t
$$

be the standard Gaussian measure on $\mathbb{R}$ and $\xi_{1}, \ldots, \xi_{n}, \ldots$ be a sequence of 
independent random variables each of which is distributed according to this Gaussian measure. Let $E \equiv\{(t, s):|t-s| \in Z\} \subset \mathbb{R}^{2}$ where the set $Z$ was defined in the proof of Theorem 4 . Then the random $\{0 ; 1\}$-matrices

$$
\left\{\chi_{Z}\left(\xi_{i}-\xi_{j}\right)\right\}_{i, j=1}^{\infty}
$$

are, with probability 1, the adjacency matrices of universal (universal trianglefree) graphs. In other words, the distribution of these random matrices is a $\mathfrak{S}^{\mathbb{N}}$-invariant measure concentrated on the universal (triangle-free) graphs. Of course, for the case of $K_{s}$-free graphs we also can choose the Gaussian measure. Instead of a Gaussian measure we can take any non-degenerate measure. The choice of the set $Z(E)$ is not unique, as follows from the construction.

\section{Classification and the complete list of in- variant measures on the set of universal graphs}

As we have seen (Corollary 1), each measurable universal graph $(X, m, E)$ produces an invariant measure on the set of universal countable graphs. Two questions arise:

1) When do two triples $(X, m, E)$ and $\left(X^{\prime}, m^{\prime}, E^{\prime}\right)$ of universal measurable graphs produce the same $\mathfrak{S}^{\mathbb{N}}$-invariant measures $\mu_{\{X, m, E\}}$ and $\mu_{\left\{X^{\prime}, m^{\prime}, E^{\prime}\right\}}$ on the set of universal graphs?

Remark, that the list of invariant measures on the set of universal countable graphs that are of type $\mu_{\{X, m, E\}}$ for some measurable universal graph $(X, m, E)$ ("randomization in vertices") is not complete. For example, it does not contain the Erdós-Rényi measure. The second question is the following:

2)How to describe the complete list of $\mathfrak{S}^{\mathbb{N}}$-invariant measures on the set of universal graphs?

We will give the answers to both questions.

\subsection{Classification of invariant measures obtained by randomization in vertices}

The answer to the first question follows from a classification theorem of [16], which claims that two pure measurable symmetric functions of two variables 
$f(x, y)$ and $f^{\prime}\left(x^{\prime}, y^{\prime}\right)$ are isomorphic ( $\Leftrightarrow$ there exists a measure-preserving map $T: X \rightarrow X^{\prime}, T m=m^{\prime}$, such that $\left.f^{\prime}(T x, T y)=f(x, y)\right)$ if and only if their matrix distributions coincide. Recall that a pure symmetric function $f(\cdot, \cdot)$ of two variables is a function for which the partition defined by the formula $\left(x \sim x_{1} \Leftrightarrow f(x, y)=f\left(x_{1}, y\right)\right.$ for almost all $\left.y\right)$ is the partition into separate points.

This property is true for a universal measurable graph. In our case, the matrix distribution in the sense of [16] is just the measure $\mu_{\{X, m, E\}}$.

Theorem 6. Two measurable universal graphs $(X, m, E)$ and $\left(X^{\prime}, m^{\prime}, E^{\prime}\right)$ produce the same measure if and only if they are isomorphic in the following sense: there exists a measure-preserving map $T:(X, m) \rightarrow\left(X^{\prime} m^{\prime}\right)$ that sends the set $E$ to $E^{\prime}$ :

$$
(x, y) \in E \Leftrightarrow(T x, T y) \in E^{\prime} .
$$

Thus the measure $\mu_{\{X, m, E\}}$ is a complete isomorphism invariant of measurable universal graphs. From this fact we immediately obtain that our construction gives uncountably many different invariant measures on the set of universal countable graphs, because even for a given topologically universal graph $(X, E)$ we can vary the measure $m$ in such a way that the measurable universal graphs $(X, m, E)$ are mutually non-isomorphic for uncountably many measures $m$. It suffices to consider $X=[0,1]$ with the Lebesgue measure $m$; then we can take uncountably many symmetric sets $E \in X^{2}$ so that the measurable functions $x \mapsto m\left(E_{x}\right)$ for different choices of $E$ have nonequal distributions as measures on $[0,1]$; these distributions are isomorphism invariants of the set $E$.

\subsection{Randomization in edges and description of the list of all invariant measures on the universal homoge- neous ( $K_{s}$-free universal homogeneous) graphs.}

In order to obtain a description of the random countable universal graph ( $K_{s}$-free-universal), or in other words invariant ergodic measure on the set of all countable universal ( $K_{s}$-free-universal) graphs), we had considered the continuous universal graph and then chose by random the vertices of the countable graph. As we already have mentioned it is impossible to obtain the list of all possible invariant measures on the set of universal graphs with 
this procedure - this "randomization in vertices" only, but another source of the randomness is "randomness in the edges", which allow to obtain whole list of invariant measures on the set of universal countable graphs. Below we explain what does this mean. But for $K_{s}$-free universal graphs we do not need the randomization in edges. We see below that it is enough to use randomization in vertices only.

Instead of the measurable graph $(X, m, E)$ we consider more general object.

Definition 5. Generalized measurable continuous graph is the triple $(X, m, \omega)$, where $(X, m)$ is the standard measure space with continuous normalized measure (Lebesgue space) and $\omega$ is any symmetric measurable function on the space $(X \times X, m \times m)$ with values $\omega(x, y) \in[0,1]$

Remark 2. In the case the function $\omega$ takes value $\{0 ; 1\}$ - (we will call this case-deterministic in edges) the subset $E=\{(x, y): \omega(x, y)=1\}$ gives the definition of the measurable graph $(X, m, E)$ in the sense of paragraph 2

The interpretation of the value of the function $\omega$ at the point $(x, y)$ is a probability that $(x, y)$ is the edge of the continuous generalized graph $(X, m, \omega)$.

\subsubsection{The list of all invariant measures for the case of universal homogeneous graphs}

Construction. This is two-step randomization. Suppose we have continuous generalized graph $(X, m, \omega)$; it produces a measure on the space of all countable graphs (or produces a random graph) as follows:

1. we choose the set of vertices $\left\{x_{k}\right\}_{k=1}^{\infty}$ as a sequence of independent random points from $X$ with respect to measure $m$ ("randomization in vertices");

2. for each chosen pairs of the vertices $\left(x_{i}, x_{j}\right)$ we define whether this pair is edge or not in our random graph independently (over all i,j) with probabilities $\omega\left(x_{i}, x_{j}\right), 1-\omega\left(x_{i}, x_{j}\right)$.

For any generalized graph $(X, m, \omega)$ this construction gives the measure, on the space of countable graphs which we denote as $M(X, m, \omega)$; will say that this measure is generated by generalized continuous graph $(X, m, \omega)$. So, we have the map from the set of the generalized graphs to the set of measures on the space of countable graphs (or its adjacent matrices).

This double randomization can be considered as randomization of the probabilities — first step, and consequent realization of those probabilities 
- the second step. Such tool is typical for the theory of the random walk in random environment.

Let us give the precise formula for the measure $M(X, m, \omega)$ of the cylindric sets. Suppose $A=\left\{a_{i, j}\right\}, i, j=1 \ldots n$ is $(0-1)$-matrix of order $n$, and $C_{A}$ is a cylindric set of all infinite $(0-1)$-matrices, which has the ma$\operatorname{trix} A$ as submatrix on the NW-corner. Then the value of the measure $M(X, m, \omega) \equiv M$ on the cylinder $C_{A}$ is

$$
M\left(C_{A}\right)=\int_{X^{n}} \prod_{(i, j): a_{i, j}=1} \omega\left(x_{i}, x_{j}\right) \prod_{(k, r): a_{k, r}=0}\left(1-\omega\left(x_{k}, x_{r}\right)\right) d m\left(x_{1}\right) \ldots d m\left(x_{n}\right) .
$$

This is a generalization of the formula given after theorem 3 in deterministic in edges case.

The following fact is evident from the definition

Theorem 7. The measure $M(X, m, \omega)$ is $\mathfrak{S}^{\mathbb{N}}$-invariant and ergodic.

Now we formulate in the convenient for the case of graphs form of the theorem by D. Aldous ([1]) which gives the description of $\mathfrak{S}^{\mathbb{N}}$-invariant measures on the space infinite matrices.

Theorem 8. Each ergodic $\mathfrak{S}^{\mathbb{N}}$-invariant measures of the set of symmetric with zero diagonal infinite $\{0 ; 1\}$-matrices is generated by generalized graph in the framework of the construction above.

Consequently, each ergodic $\mathfrak{S}^{\mathbb{N}}$-invariant measures on the set of all countable graphs can be obtained by or construction above. We will not discuss here the proof of Aldous theorem but remark that the second author will present elsewhere an alternative approach ("ergodic method") to this theorem and will give a new proof of it (see also discussion in [16]).

Remark also, that randomization in edges (when exist) cannot be reduced to randomization in vertices: more exactly, the resulting measure $M(X, m, \omega)$ can not be obtained as a measure corresponding to the measurable graph $(X, m, E) 9$.

Now we must formulate the special condition on the generalized graph when the construction above gives the measure on the universal $\left(K_{s}\right.$-free universal) homogeneous graph. The condition is similar to the condition of the theorem 2. We will formulate it only for universal graphs: for $K_{s}$-free universal graphs $s>2$ we do not need such notion (see below).

\footnotetext{
${ }^{9}$ It corresponds in a sense to the generalized function $\omega$ (see [16])
} 
Definition 6. The generalized measurable graph $(X, m, \omega)$ is called universal if for all natural $n, m$ and almost all pairs of the sets $\left(x_{1}, \ldots, x_{n}\right),\left(y_{1}, \ldots, y_{m}\right)$ from $X$ the following is true

$$
m\left\{z \in X: \prod_{i, j} \omega\left(x_{i}, z\right)\left(1-\omega\left(y_{j}, z\right)\right)>0\right\}>0 .
$$

Here were restrict ourselves with the following important remark. The first step of the construction gives us a family Bernoulli measures on the 0-1-matrices (all entries are independent but have in general different distributions) and the resulting measure on the space of $0-1$-matrices is the average (more exactly barycenter) of the those Bernoulli measures.

The explicit construction of the universal ( $K_{s}$-free universal) generalized graph can be done (even simpler) as for universal topological graph in the section 3 which is of course partial case. We will not consider this, but emphasize that the constructions of the section 3 have more instructive character than construction of generalized universal graph.

Now we can give a list of all $\mathfrak{S}^{\mathbb{N}}$-invariant ergodic measures on the space of all universal countable graphs (or on the set of corresponding 0; 1 matrices), which is the mail goal of this section. This is the corollary of the previous theorems:

Theorem 9. Each ergodic $\mathfrak{S}^{\mathbb{N}}$-invariant measures on the set of all countable universal homogeneous graphs is generated by the construction above with generalized measurable universal graph.

\subsubsection{The list of measures for $K_{s}$-free universal homogeneous graphs.}

Theorem 10. The list of all invariant ergodic measures on the $K_{s}$-free (for $s>2$ ) countable homogeneous graphs is given by randomization in vertices only, e.f. by the construction of theorem 3 of the subsection 2.2. In other words, in order to obtain whole list it is enough to use only the randomization in vertices of measurable graph and no randomization in edges.

We outline the simple proof of this fact. Let for simplicity consider the case $s=3$; the general case is analogous. Assume the contrary, and suppose that there exist a set of positive measure $F \subset X^{2}$ such that for $(a, b) \in$ $F, 0<\epsilon_{1}<\omega(a, b)<\epsilon_{2}<1$. Then on the one hand, $a$ and $b$ have almost no common neighbor for almost all $(a, b) \in B$ (since the graph must be trianglefree), on the other hand $a$ and $b$ for almost all $(a, b) \in B$ must have a common 
neighbor with positive probability (since the graph must be universal, and if we do not take the edge a-b, then these vertices should have some common neighbor).

\section{Some problems and comments}

1. The distribution of the entries of random adjacency. A very important question is to characterize, for an arbitrary invariant measure $M(X, m, \omega)$ (see the previous section), the finite-dimensional distribution of the entries with respect to this measure. Because of the $\mathfrak{S}^{\mathbb{N}}$-invariance of the measure, the finite-dimensional distributions are $\mathfrak{S}_{n}$-invariant; consequently, they are concentrated on bunches of orbits of these groups and decompose into positive combinations of orbits of the group $\mathfrak{S}_{n}$ in the space of matrices $M_{n}(0,1)$. Perhaps, because of the ergodicity of the measure $M(X, m, \omega)$, the finite-dimensional distributions must concentrate near one or several typical orbits at short distances from one another. This is an analog of the Law of Large Numbers. How to characterize these orbits? The answer could be useful for the solution of the problem by Cherlin (see introduction). The structure and the asymptotic size of these orbits is an interesting characteristic of universal graphs and the measures.

2. Uniqueness of measurable universal graphs. When discussing the definitions of universality above, the following question naturally arises: under what conditions is the set $E$ which defines the graph structure on the standard Borel (or standard measure) space of universal Borel or measurable graphs unique up to isomorphism? In the case of countable universal graphs, the "back and forth" method allows one to prove the uniqueness of the universal graph. Equivalently, the question above is as follows: when does the Borel or measurable version of the "back and forth" method work? The same question can be solved positively for metric spaces: as proved by Urysohn, there exists a unique (up to isomorphism) universal Polish space. It is interesting to have link with model theory in which one consider finite or countable situation. When uniqueness takes place in the continuous case?

3. Approximation. In our construction we obtained a continuous graph with $\mathbb{R}$ as the set of vertices as the completion of a graph with the set of vertices $\mathbb{Q}$. Of course, in that case we could define the graph directly, avoiding approximation. But it is interesting whether in the general situation of model theory it is possible to consider a "completion" of countable models. 
More exactly, how to formulate Fraïssé's axioms for the Borel or measurable case (with separability conditions) in order to obtain it as the projective or another limit of the finite theory? A very good example of a positive solution of such a problem is, of course, the theory of universal metric spaces.

4. Link to the Urysohn space. In this sense, the Urysohn space is of special interest. We will consider it from this point of view elsewhere. Here we mention only that the Urysohn space $\mathbb{U}$ plays the role of a "Borel universal object" (or topologically universal object) for the rational or integer universal metric space. Any Borel probability measure $m$ on this space defines a $\mathfrak{S}^{\mathbb{N}}$-invariant measure $\mu$ on the space $\mathcal{R}$ of distance real matrices which are universal10 with probability one. The similarity between the theory of the Urysohn space and the example of Section 3 above can be illustrated by the result of [4] where the Urysohn space was realized as the completion of the real line with respect to a universal shift-invariant metric.

\section{References}

[1] D. Aldous. Representations for partially exchangeable arrays of random variables. J. Multiv. Anal. 11, 581-598 (1981).

[2] P. Cameron. The random graph. In: The Mathematics of Paul Erdos (R. L. Graham and J. Nesetril, eds.), Springer, Berlin, 1997, pp. 331351.

[3] P. Cameron. Homogeneous Cayley objects. Europ. J. Combin. 21, No. 6, 745-760 (2000).

[4] P. Cameron and A. Vershik. Some isometry groups of Urysohn spaces. Ann. Pure Appl. Logic 143, No. 1-3, 70-78 (2006).

[5] P. Diaconis and S. Janson. Graph limits and exchangeable random graphs. arXiv:0712.2749v1 (2007).

[6] P. Erdös, D. J. Kleitman, and R. Rothschild. Asymptotic enumeration of $K_{n}$-free graphs. Colloquio Internazionale sulle Teorie Combinatorie (Rome, 1973), Tomo II, pp. 19-27. Atti dei Convegni Lincei, No. 17, Accad. Naz. Lincei, Rome, 1976.

\footnotetext{
${ }^{10}$ The universality of a distance matrix means that the completion of $\mathbb{N}$ with respect to the corresponding metric is isometric to the Urysohn space, see [18]
} 
[7] P. Erdös and A. Rényi. Asymmetric graph. Acta Math Acad. Sci. Hungar. 14, 295-315 (1963).

[8] C. W. Henson. A Family of Countable Homogeneous Graphs. Pacific Journ.Math. 38, No.1, 69-83 (1971).

[9] W. Hodges. A Shorter Model Theory. Cambridge Univ. Press, Cambridge, 1997.

[10] O. Kallenberg. Probabilistic Symmetries and Invariance Principles. Springer, New York, 2005.

[11] Ph. Kolaitis, H. Prömel, and B. Rothschild. $K_{l+1}$-free graphs: asymptotic structure and a 0-1 law. Trans. Amer. Math. Soc. 303, No. 2, 637-671 (1987).

[12] L. Lovasz. The rank connection matrices and the dimension of graph algebras. Europ. J. Combin. 27, 962-970 (2006).

[13] L. Lovasz and B. Szegedy. Limits of dense graph sequences. J. Combin. Theory B 96, No. 6, 933-957 (2006).

[14] R. Rado. Universal graph and universal functions. Acta Arithm. 9, 331340 (1964).

[15] P. Urysohn. Sur un espace metrique universel. Bull. Soc. Math. 51, 1-38 (1927).

[16] A. Vershik. Classification of measurable functions of several arguments, and invariantly distributed random matrices. Funct. Anal. Appl. 36, No. 2, 93-105 (2002).

[17] A. Vershik. Random metric spaces and universality. Russian Math. Surveys 59, No. 2, 259-295 (2004).

[18] A. Vershik. Kolmogorov's example (a survey of actions of infinitedimensional groups with invariant measure). Theory Probab. Appl. 48, No. 2, 373-378 (2004).

[19] A. Vershik. Globalization of partial isometries of metric spaces and local approximation of the group of isometries of the Urysohn space. Topology and its Appl. 155, No. 14, 1618-1626, (2008). 